\title{
Relationship between the Kubelka-Munk scattering and radiative transfer coefficients
}

\author{
Suresh N. Thennadil \\ School of Chemical Engineering and Advanced Materials, University of Newcastle upon Tyne, \\ Newcastle upon Tyne, NE1 7RU, United Kingdom \\ s.n.thennadil@ncl.ac.uk
}

The relationship between the Kubelka-Munk (K-M) scattering and the transport scattering coefficient is obtained through a semi-empirical approach. This approach gives the same result as that given by Gate when the incident beam is diffuse. This result and those given by Star et al and Brinkworth are compared with the exact solution of the radiative transfer equation over a large range of optical properties. It is found that the latter expressions which include an absorption component do not give accurate results over the range considered. Using the semiempirical approach, the relationship between the K-M and transport scattering coefficient is derived for the case where the incident light is collimated. It is shown that although the K-M equation is derived based on diffuse incident light, it can also represent very well the reflectance from a slab of infinite thickness when the incident light is collimated. However, in this case the relationship between the coefficients has to include a function that is dependent on the anisotropy factor. Analysis indicates that the K-M transform achieves the objective of obtaining a measure that gives the ratio of absorption to scattering effects for both diffuse and collimated incident beam over a large range of optical properties. (C) 2007 Optical Society of America

OCIS codes: $010.5620,290.4210,290.7050,290.5850$. 


\section{Introduction}

Diffuse reflectance spectroscopy has been used as a characterisation tool for a wide variety of applications involving particulate media such as paints, films and powders. The phenomenological Kubelka-Munk (K-M) theory has been extensively used for analysing reflectance spectra of such media [1-6]. While light propagation can be more rigorously described using the radiative transfer equation (RTE), the computational complexity of the RTE especially when the exact solution is invoked makes it hard to use under practical conditions. Although approximate expressions derived from the RTE have been available, the simplicity of the K-M theory with its simple analytical solution has been the main reason for its continued widespread use. According to this theory [1], the reflectance from a slab of infinite depth $R_{\infty}$ is given by:

$$
\mathrm{R}_{\infty, \mathrm{KM}}=1+\frac{\mathrm{K}}{\mathrm{S}}-\sqrt{2}\left(\frac{\mathrm{K}}{\mathrm{S}}\right)^{1 / 2}\left(1+\frac{\mathrm{K}}{2 \mathrm{~S}}\right)^{1 / 2}
$$

Particularly, the Kubelka-Munk transform for converting the reflectance from a semi-infinite slab into a ratio of "absorption" and "scattering" effects has been widely used for analysing such samples [4-6]:

$$
\frac{\mathrm{K}}{\mathrm{S}}=\frac{\left(1-\mathrm{R}_{\infty}\right)^{2}}{2 \mathrm{R}_{\infty}}
$$

The assumption underlying the use of this transform for analysing reflectance data is that the phenomenological absorption and scattering coefficients are functions of purely the physical absorption and scattering coefficients respectively and thus their ratio is proportional to the corresponding ratio of the physical optical properties. However, the K-M theory does not provide 
an explicit connection between K-M coefficients and the optical properties of individual particles as can be obtained in the case of the RTE based formulas. One approach to obtain this connection is to establish the relationship between the transport coefficients from the RTE and the $\mathrm{K}-\mathrm{M}$ coefficients.

The relationship between K-M and the transport coefficients have been examined previously by several researchers [7-9] who used the radiative transfer equation (RTE) as the starting point. In these cases the relationship between the Kubelka-Munk absorption coefficient $\mathrm{K}$ and the transport absorption coefficient $\mu_{\mathrm{a}}$ was given by

$$
\mathrm{K}=2 \mu_{\mathrm{a}}
$$

For the scattering coefficients slightly different relationships were obtained by different researchers with the general form:

$$
\mathrm{S}=\mathrm{y} \mu_{\mathrm{s}}^{\prime}-\mathrm{x} \mu_{\mathrm{a}}
$$

where $\mathrm{S}$ is the Kubelka-Munk scattering coefficient, $\mu_{\mathrm{S}}^{\prime}=\mu_{\mathrm{S}}(1-\mathrm{g})$ is the reduced transport scattering coefficient, $\mu_{\mathrm{s}}$ is the transport scattering coefficient and $\mathrm{g}$ is the anisotropy factor. Brinkworth's formula [7] is obtained when $\mathrm{x}=1$, Gate's formula [8] is given by $\mathrm{x}=0$ and Star $e t$ al [9] give $\mathrm{x}=1 / 4$. All of them give $\mathrm{y}=3 / 4$. This study focuses on obtaining a relationship between $\mathrm{S}$ and the transport coefficients through a semi-empirical approach. The relationship between $\mathrm{K}$ and the transport coefficients is taken to be the same as that given by (3). 
According to (3) and (4), the ratio of $\mathrm{K} / \mathrm{S}$ does not strictly provide a ratio of purely absorption and scattering effects since $\mathrm{S}$ contains absorption effects in addition to scattering except when $\mathrm{x}$ $=0$ i.e. when Gate's formula is used. Examining (4), it can be seen that this equation can at best be only valid when $\mu_{\mathrm{s}}^{\prime}>(\mathrm{x} / \mathrm{y}) \mu_{\mathrm{a}}$. When $\mu_{\mathrm{s}}^{\prime}$ is equal to or less than this value we get physically unrealistic values for $S$ and consequently for $R_{\infty}$ calculated using (1). As a result, it is not clear whether including the second term in (4) gives an accurate relationship over a range of values of $\mu_{\mathrm{a}}$ and $\mu_{\mathrm{s}}^{\prime}$ to be of practical use.

A plausible alternative is to use a simplified formula for diffuse reflectance which gives reasonably good estimates and from which we can derive the ratio of the physical absorption and scattering effects i.e. $\mu_{\mathrm{a}} / \mu_{\mathrm{s}}^{\prime}$ in terms of $\mathrm{R}_{\infty}$. A semi-empirical formula based on the transport coefficients was proposed by Jacques [10] where the reflectance from a semi-infinite media is approximately represented by a Beer's law type of equation:

$$
\mathrm{R}_{\infty}=\exp \left(-\mathrm{C}_{0} \mu_{\mathrm{a}} \delta\right)
$$

Here $\mathrm{R}_{\infty}$ is the total diffuse reflectance, $\mathrm{C}_{0}$ is a constant and $\delta$ is the penetration depth given by:

$$
\delta=\left[3 \mu_{\mathrm{a}}\left(\mu_{\mathrm{a}}+\mu_{\mathrm{s}}^{\prime}\right)\right]^{-0.5}
$$

The constant $C_{0}$ can be obtained by fitting $R_{\infty}$ obtained for known values of $\mu_{\mathrm{a}}$ and $\mu_{\mathrm{s}}^{\prime}$. 
In this paper, the range of validity of (5) is examined by using data generated by solving the exact RTE using the doubling method for diffuse and collimated incident beams [11]. It is then shown that when $\mu_{\mathrm{a}} / \mu_{\mathrm{s}}^{\prime}<<1$ (the region of validity of (5)), the K-M equation for reflectance from a slab of infinite thickness and (5) are equivalent. From this comparison, the value of y in (4) is obtained for the cases of diffuse and collimated incident beam. The relationship so obtained is compared with those reported by the researchers mentioned earlier. The approach is then applied to collimated incident beam to investigate the possibility of using the K-M equation under such condition.

\section{Data set generation}

The RTE was solved using the doubling method implemented in Matlab [11-12]. For this study matched boundary conditions were used i.e. the refractive index of the sample and the surrounding medium are the same. The simulations were carried out for transport parameters generated using a full factorial design with the following range of parameters: $\mu_{\mathrm{a}}=0.02-15$ $\mathrm{mm}^{-1} ; \mu_{\mathrm{s}}=0.2-40 \mathrm{~mm}^{-1}$ and $\mathrm{g}=0.001-0.95$. Data for these values were generated for two types of incident beams: Diffuse and collimated. For both these cases, normal incidence was assumed.

\section{Results and discussions}

\subsection{Validity of Eq. (5)}

According to (5), the logarithm of $\mathrm{R}_{\infty}$ should be linear in $\mu_{\mathrm{a}} \delta$. From figure 1a, which shows the results for the case of diffuse incident beam, it can be seen that the relationship becomes highly nonlinear beyond $\mu_{\mathrm{a}} \delta$ of about 0.4 . In fact, further examination showed the relationship can be 
reasonably approximated by the linear relationship for $\mu_{\mathrm{a}} \delta \leq 0.18$ as shown in figure $1 \mathrm{~b}$. The residuals plot figure $1 \mathrm{c}$, shows the error in using (5) to compute $\mathrm{R}_{\infty}$ for this range. It can be seen that there is still a nonlinear component which is not explained. However, for this range of $\mu_{\mathrm{a}} \delta$ values, this unexplained component is very small - in the order of $10^{-3}$. This translates to less than $1 \%$ error in the calculated reflectance over the range of optical properties considered (with an RMS error of about $0.5 \%$ over this range). In terms of $\mu_{\mathrm{a}} / \mu_{\mathrm{s}}^{\prime}$, this upper limit of $\mu_{\mathrm{a}} \delta$ translates to a value of about 0.1 . This is arrived at from the relationship derived using (6):

$$
\frac{\mu_{\mathrm{a}}}{\mu_{\mathrm{s}}^{\prime}}=\frac{3\left(\mu_{\mathrm{a}} \delta\right)^{2}}{1-3\left(\mu_{\mathrm{a}} \delta\right)^{2}}
$$

Fitting (5) to the reflectance data for this range of $\mu_{\mathrm{a}} / \mu_{\mathrm{s}}^{\prime}$, for the case of diffuse incident beam, we get $\mathrm{C}_{0}=4.0$.

When the incident beam is collimated, figure 2(a) shows similar characteristics in terms of linearity as for the case of diffuse incident beam. However, the "spread" in the reflectance due to the variations in $g$ at each value of $\mu_{\mathrm{a}} \delta$ is significantly higher indicating a stronger dependence on $g$ compared to the case of diffuse incident beam where this effect was negligible. Figure 2(b) shows a plot of $R_{\infty}$ in the linear range of $\mu_{\mathrm{a}} \delta$ for two extreme values of $g(=0.001$ and 0.95$)$. It can be seen that the effect of $g$ is essentially on the slope and thus $C_{0}$ can be expected to be a function of $\mathrm{g}$. The variation of $\mathrm{C}_{0}$ with $\mathrm{g}$ (Figure $2 \mathrm{c}$ ) was found by estimating $\mathrm{C}_{0}$ using reflectance data from solving the RTE with g fixed at different values. The solid line in Figure 2c was obtained by fitting a second degree polynomial: 


$$
\mathrm{C}_{0}(\mathrm{~g})=4.8446+0.472 \mathrm{~g}-0.114 \mathrm{~g}^{2}
$$

It can be seen that variation of $\mathrm{C}_{0}$ with $\mathrm{g}$ can be well represented by a second order polynomial.

\subsection{Comparison of Eq. 5 with K-M equation}

Eq. (7), can be re-written as:

$$
\mu_{\mathrm{a}} \delta=\frac{\sqrt{\mu_{\mathrm{a}} / \mu_{\mathrm{s}}^{\prime}}}{\sqrt{3\left(1+\mu_{\mathrm{a}} / \mu_{\mathrm{s}}^{\prime}\right)}}
$$

When $\mu_{\mathrm{a}} / \mu_{\mathrm{s}}^{\prime}<<1$, this reduces to:

$$
\mu_{\mathrm{a}} \delta \approx \frac{\sqrt{\mu_{\mathrm{a}} / \mu_{\mathrm{s}}^{\prime}}}{\sqrt{3}}
$$

Substituting in Eq. 5, we get

$$
\mathrm{R}_{\infty}=\exp \left(-\frac{\mathrm{C}_{0}}{\sqrt{3}} \sqrt{\frac{\mu_{\mathrm{a}}}{\mu_{\mathrm{s}}^{\prime}}}\right)
$$

Using the series expansion of the right hand side and neglecting powers higher than $1,(11)$ becomes

$$
\mathrm{R}_{\infty}=1-\frac{\mathrm{C}_{0}}{\sqrt{3}}\left(\frac{\mu_{\mathrm{a}}}{\mu_{\mathrm{s}}^{\prime}}\right)^{1 / 2}+\frac{1}{2}\left(\frac{\mathrm{C}_{0}}{\sqrt{3}}\right)^{2} \frac{\mu_{\mathrm{a}}}{\mu_{\mathrm{s}}^{\prime}}
$$

The Kubelka-Munk equation is derived under the conditions that the incident beam is diffuse and that scattering is isotropic and absorption effects are small compared to scattering. For a slab of 
infinite thickness, the reflectance according to the K-M theory is given by (1). Using the Binomial theorem,

$$
\left(1+\frac{\mathrm{K}}{2 \mathrm{~S}}\right)^{1 / 2}=1+\frac{1}{2} \frac{\mathrm{K}}{2 \mathrm{~S}}+\ldots
$$

Substituting (13) in (1) and neglecting powers of K/S greater than 1, we get,

$$
\mathrm{R}_{\infty, \mathrm{KM}}=1-\sqrt{2}\left(\frac{\mathrm{K}}{\mathrm{S}}\right)^{1 / 2}+\frac{\mathrm{K}}{\mathrm{S}}
$$

From (3) and (4), we get

$$
\frac{\mathrm{K}}{\mathrm{S}}=\frac{2 \mu_{\mathrm{a}}}{\mathrm{y} \mu_{\mathrm{s}}^{\prime}-\mathrm{x} \mu_{\mathrm{a}}}
$$

Under the condition $\mu_{\mathrm{a}} / \mu_{\mathrm{s}}^{\prime}<<1$ this becomes,

$$
\frac{\mathrm{K}}{\mathrm{S}}=\frac{2}{\mathrm{y}} \frac{\mu_{\mathrm{a}}}{\mu_{\mathrm{S}}^{\prime}}
$$

It should be pointed out that the same relationship can be obtained by simply setting $\mathrm{x}=0$ in (4) instead of invoking the condition $\mu_{\mathrm{a}} / \mu_{\mathrm{s}}^{\prime}<<1$. Due to the problem of physically unrealistic values occurring if the second term in (4) is used (as was mentioned in the introduction), $x=0$ is assumed in this analysis and the results are compared with those obtained when $x \neq 0$ i.e. the relationships given by Star et al and Brinkworth. Substituting for K/S using (16), Eq. (14) becomes 


$$
\mathrm{R}_{\infty, \mathrm{KM}}=1-\frac{2}{\sqrt{\mathrm{y}}}\left(\frac{\mu_{\mathrm{a}}}{\mu_{\mathrm{s}}^{\prime}}\right)^{1 / 2}+\frac{2}{\mathrm{y}} \frac{\mu_{\mathrm{a}}}{\mu_{\mathrm{s}}^{\prime}}
$$

Comparing the coefficients of (12) and (17) term by term,

$$
\frac{\mathrm{C}_{0}}{\sqrt{3}}=\frac{2}{\sqrt{\mathrm{y}}} \text { and } \quad \frac{\mathrm{C}_{0}^{2}}{6}=\frac{2}{\mathrm{y}}
$$

From which we get,

$$
\mathrm{y}=\frac{12}{\mathrm{C}_{0}^{2}}
$$

Substituting in (16),

$$
\frac{\mathrm{K}}{\mathrm{S}}=\frac{\mathrm{C}_{0}^{2}}{6} \frac{\mu_{\mathrm{a}}}{\mu_{\mathrm{s}}^{\prime}}
$$

Also, from (4) with $\mathrm{x}=0$ and using (19) we get,

$$
\mathrm{S}=\frac{12}{\mathrm{C}_{0}^{2}} \mu_{\mathrm{s}}^{\prime}
$$

When the incident light is diffuse, as discussed in the previous section, the value of $\mathrm{C}_{0}$ was found to be 4.0. Substituting in (19), we get $y=3 / 4$ and from (21) we get,

$$
\mathrm{S}=\frac{3}{4} \mu_{\mathrm{S}}^{\prime}
$$

and 


$$
\frac{\mathrm{K}}{\mathrm{S}}=\frac{8}{3} \frac{\mu_{\mathrm{a}}}{\mu_{\mathrm{S}}^{\prime}}
$$

This corresponds to the relationship obtained by Gate. Thus, the process of obtaining (19) is validated using the case of diffuse incident beam.

The question that arises now is whether this relationship between the K-M and the transport scattering coefficients holds beyond the limiting range of $\mu_{\mathrm{a}} / \mu_{\mathrm{s}}^{\prime}<<1$ from which it was derived. To investigate this, $\mathrm{R}_{\infty}$ calculated using the exact RTE for the range of optical properties specified in section 2 were compared with that obtained from the K-M equation after estimating the K-M coefficients using (3) and (22) and also by those given by Star et. al. and Brinkworth. The range of optical properties considered translates to $\mu_{\mathrm{a}} / \mu_{\mathrm{s}}^{\prime} \leq 75$ which in turn translates to $\mu_{\mathrm{a}} \delta \leq 0.577$. From figure 3(a), it is seen that for this wide range of optical properties, (22) which is the same as the expression given by Gate - provides the best agreement with the exact values of $\mathrm{R}_{\infty}$. The extra term contained in the formulas of Brinkworth and Star et. al, which includes a contribution from $\mu_{\mathrm{a}}$ to the KM scattering coefficient (Eq. 4), actually results in a deviation from the RTE especially with increasing values of $\mu_{\mathrm{a}}$ and as discussed earlier, it leads to singularities for certain combinations of the optical properties. From figure 3(b) it is seen that the error in using the K-M equation with S given by (22) leads to an error of $<3 \%$ over the range of optical properties considered.

In the section 3.1 it was shown that the value of $\mathrm{C}_{0}$ depends on the nature of the incident beam and when the incident beam is collimated $\mathrm{C}_{0}$ is a function of $\mathrm{g}$ which can be empirically 
described by a second-order polynomial. Thus, by substituting the g-dependent form of $\mathrm{C}_{0}$ given by (8) into (21) we obtain the relationship between the K-M scattering coefficient and the transport scattering coefficient for the case when the incident beam is collimated:

$$
\mathrm{S}=\frac{12}{\left(4.8446+0.472 \mathrm{~g}-0.114 \mathrm{~g}^{2}\right)^{2}} \mu_{\mathrm{S}}^{\prime}
$$

In this case, (20) can be rewritten as

$$
\frac{K}{S}=\frac{\mu_{a}}{\mu_{s}^{\prime} f(g)}
$$

where

$$
f(g)=\frac{6}{C_{0}^{2}(g)}
$$

$\mathrm{C}_{0}(\mathrm{~g})$ is given by (8). Eq. 25 indicates that for the case of collimated incident beam just as for the diffuse incident beam, the ratio K/S still corresponds to a ratio of absorption to scattering effects except that the functional dependence on $\mathrm{g}$ for the scattering component (denominator) is different.

Figure 4 shows a comparison of $\mathrm{R}_{\infty}$ computed with the exact RTE solved using the doubling method when the incident beam is collimated with those computed from the K-M equation with $\mathrm{S}$ given by (24). For comparison purposes, $\mathrm{R}_{\infty}$ predicted by the K-M equation using the relationship (22) which was derived for diffuse incident beam is also plotted. In figure 4, the comparisons are shown for a low $(\mathrm{g}=0.1)$ and a high $(\mathrm{g}=.9)$ value of $\mathrm{g}$. It is seen that $(24)$ 
shows excellent agreement with the exact values of $R_{\infty}$. The residuals plots, figures $4(c)$ and $(d)$ show that when the $\mathrm{g}$ dependence is taken into account the error is less than $5 \%$ over most of range considered. The errors increase at the higher range but still stay within $10 \%$. This indicates that the form given by (20) also holds for collimated incident beam as long as the dependence of $\mathrm{C}_{0}$ on $\mathrm{g}$ is taken into account.

In practice, even though the K-M equations are derived for the specific case of diffuse incident beam of infinite width, the K-M transform (Eq. 2) has been applied for analysing spectra even when the incident beam is not diffuse. The foregoing analysis indicates that the K-M transform achieves the objective of obtaining a measure that gives the ratio of absorption to scattering effects for both diffuse and collimated incident beam.

\section{Conclusions}

In this manuscript, the relationship between the K-M scattering and the transport scattering coefficient was obtained through a semi-empirical approach. This approach gives the same result as that given by Gate when the incident beam is diffuse. It is shown that although the K-M equation is derived based on diffuse incident light, it can also represent very well the reflectance from a slab of infinite thickness when the incident light is collimated. However, in this case the relationship between $\mathrm{S}$ and $\mu_{\mathrm{S}}^{\prime}$ has to include a function that is dependent on $\mathrm{g}$. This study also shows that the relationships given by Star et al and Brinkworth where S contains the effect of $\mu_{\mathrm{a}}$ is not applicable over a large range of optical properties. This study suggests that the Kubelka-Munk transform achieves the objective of obtaining a measure that gives the ratio of absorption to scattering effects for both diffuse and collimated incident beam. 


\section{Acknowledgments}

This work was funded by the EPSRC through grants GR/S50441/01 and GR/S50458/01.

\section{References:}

1. G. Kortüm, Reflectance Spectroscopy (Springer-Verlag, 1969).

2. M. K. Gunde, J. K. Logar, Z. C. Orel and B. Orel, "Application of the Kubelka-Munk theory to thickness-dependent diffuse reflectance of black paint in the mid-IR," Appl. Spec. 49, 623629 (1995).

3. L. E. McNeil and R. H. French, "Light scattering from red pigment particles: Multiple scattering in a strongly absorbing system," J. Appl. Phys. 89, 283-293 (2001).

4. G. Dupuis and M. Menu, "Quantitative characterisation of pigment mixtures used in art by fibre-optics diffuse-reflectance spectroscopy,” Appl. Phys. A 83, 469-474 (2006).

5. J. Sirita, S. Phanichphant and F. C. Meunier, "Quantitative analysis of adsorbate concentrations by diffuse reflectance FT-IR,” Anal. Chem. 79, 3912-3918 (2007).

6. P. Jeevanandam, R. S. Mulukutla, M. Phillips, S. Chaudhuri, L. E. Erickson and K. J. Klabunde, "Near infrared reflectance properties of metal oxide particles," J. Phys. Chem. 111, 1912-1918 (2007).

7. B. J. Brinkworth, "Interpretation of the Kubelka-Munk coefficients in reflection theory," Appl. Opt. 11, 1434-1435 (1972).

8. L. F. Gate, "Comparison of the photon diffusion model and Kubelka-Munk equation with the exact solution of the radiative transport equation," Appl. Opt. 13, 236-238 (1974). 
9. W. M. Star, J. P. A. Marijnissen and M. J. C. Van Gemert, "Light dosimetry in optical phantoms and in tissues: I. Multiple flux and transport theory," Phys. Med. Biol. 33, 437-454 (1988).

10. S.L. Jacques, "Reflectance spectroscopy with optical fiber devices, and transcutaneous bilirubinometers,"Biomedical optical instrumentation and laser-assisted Biotechnology, Proceedings of the NATO Advanced Science Institute (Erice, Sicily, Kluwer Academic Publishers, Nov 10-22, 1995), eds. A.M. Verga and Scheggi.

11. S. A. Prahl, "The Adding-Doubling Method," in Optical Thermal Response of Laser Irradiated Tissue, A. J. Welch, and v. G. M. J. C., eds. Plenum Press, New York, (1995), pp. $101-129$.

12. M. A. Velazco-Roa, and S. N. Thennadil, "Estimation of complex refractive index of polydisperse particulate systems from multiple-scattered ultraviolet-visible-near-infrared measurements" Applied Optics 46 3730-3735 (2007). 


\section{List of Figures:}

Figure1. Reflectance from a slab of infinite thickness irradiated by diffuse incident beam as a function of $\mu_{\mathrm{a}} \delta:$ (a) $\mathrm{R}_{\infty}$ over the whole range considered in this study. (b) Comparison of $\mathrm{R}_{\infty}$ calculated using Eq.(5) in the "linear" region. (c)Error in using Eq.(5) in the "linear" region.

Figure2. Reflectance from a slab of infinite thickness irradiated by collimated incident beam as a function of $\mu_{\mathrm{a}} \delta$ : (a) $\mathrm{R}_{\infty}$ over the whole range considered in this study. (b) $\mathrm{R}_{\infty}$ for two (low and high) values of $\mathrm{g}$ in the "linear" region. (c) $\mathrm{C}_{0}$ as a function of $g$ estimated by fitting Eq. (5) to exact values at different $g$.

Figure3. Comparison of results for diffuse incident beam using K-M scattering coefficient $\mathrm{S}$ computed with relationships given by Star et.al, Brinkworth and the present study. (a) $\mathrm{R}_{\infty}$ from the K-M equation using the various relationships is compared to the exact RTE. (b) Relative error of the K-M equation when (21) is used to related S with $\mu_{\mathrm{S}}^{\prime}$.

Figure4. Comparison of results for collimated incident beam using K-M scattering coefficient S computed with (22) and (24). (a) $\mathrm{R}_{\infty}$ when $\mathrm{g}=0.1$; (b) $\mathrm{R}_{\infty}$ when $\mathrm{g}=0.9$; (c) Relative error when $\mathrm{g}=0.1$ and $(\mathrm{d})$ Relative error when $\mathrm{g}=0.9$. 

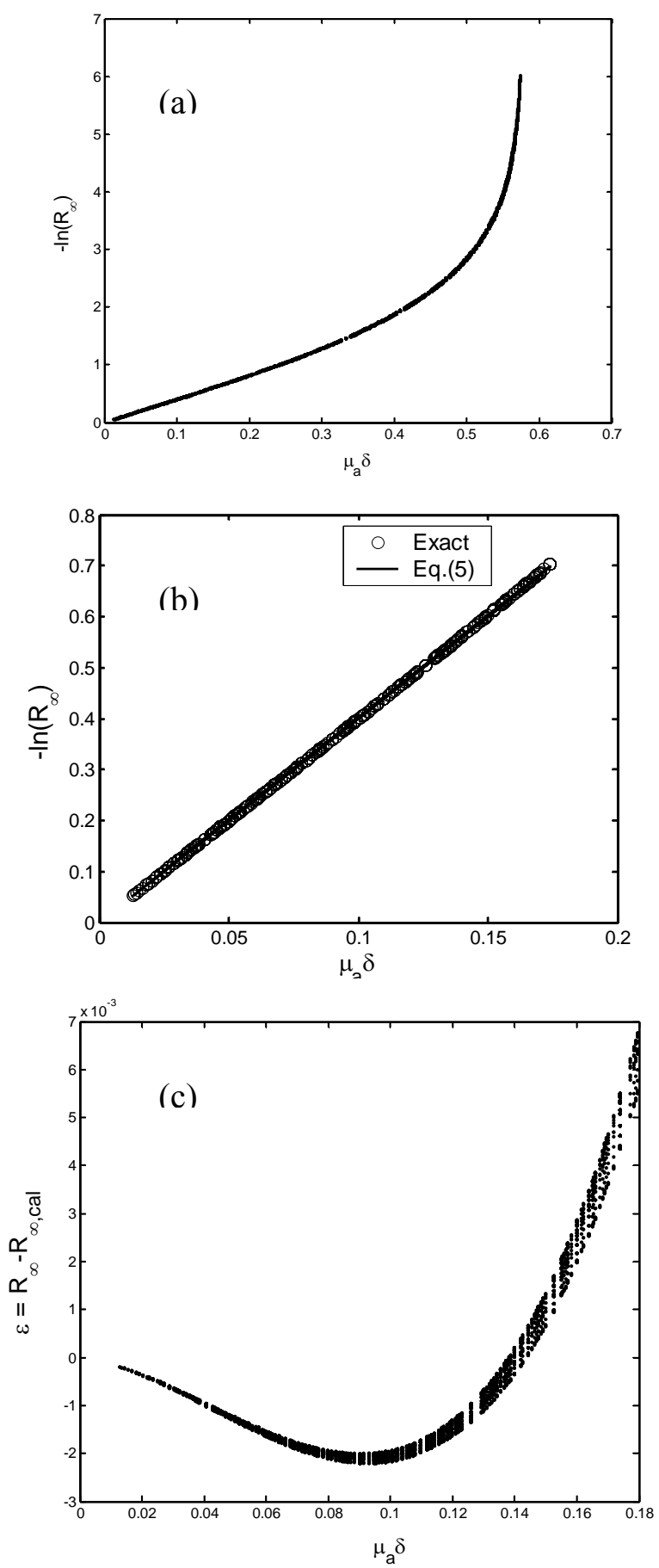

Figure1. Reflectance from a slab of infinite thickness irradiated by diffuse incident beam as a function of $\mu_{\mathrm{a}} \delta$ : (a) $\mathrm{R}_{\infty}$ over the whole range considered in this study. (b) Comparison of $\mathrm{R}_{\infty}$ calculated using Eq.(5) in the "linear" region. (c)Error in using Eq.(5) in the "linear" region. 

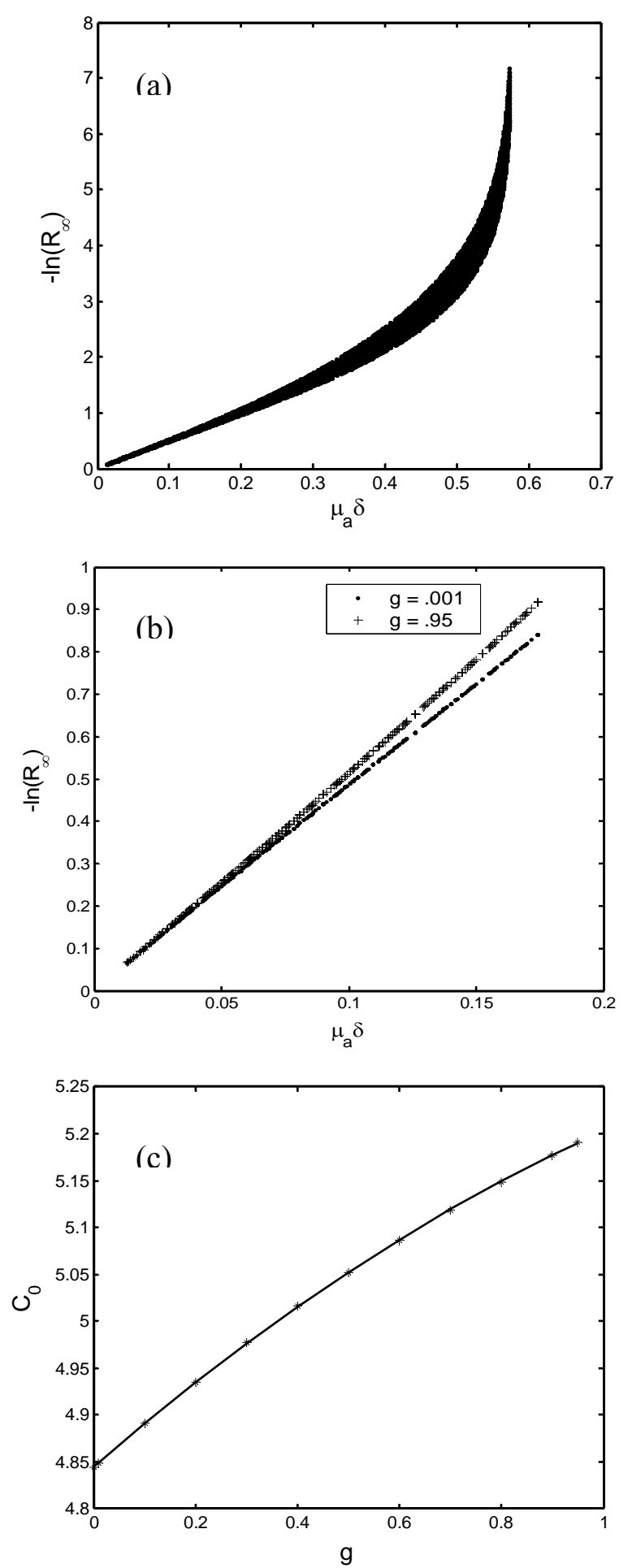

Figure2. Reflectance from a slab of infinite thickness irradiated by collimated incident beam as a function of $\mu_{\mathrm{a}} \delta$ : (a) $\mathrm{R}_{\infty}$ over the whole range considered in this study. (b) $\mathrm{R}_{\infty}$ for two (low and high) values of $\mathrm{g}$ in the "linear" region. (c) $\mathrm{C}_{0}$ as a function of $\mathrm{g}$ estimated by fitting Eq. (5) to exact values at different $g$. 

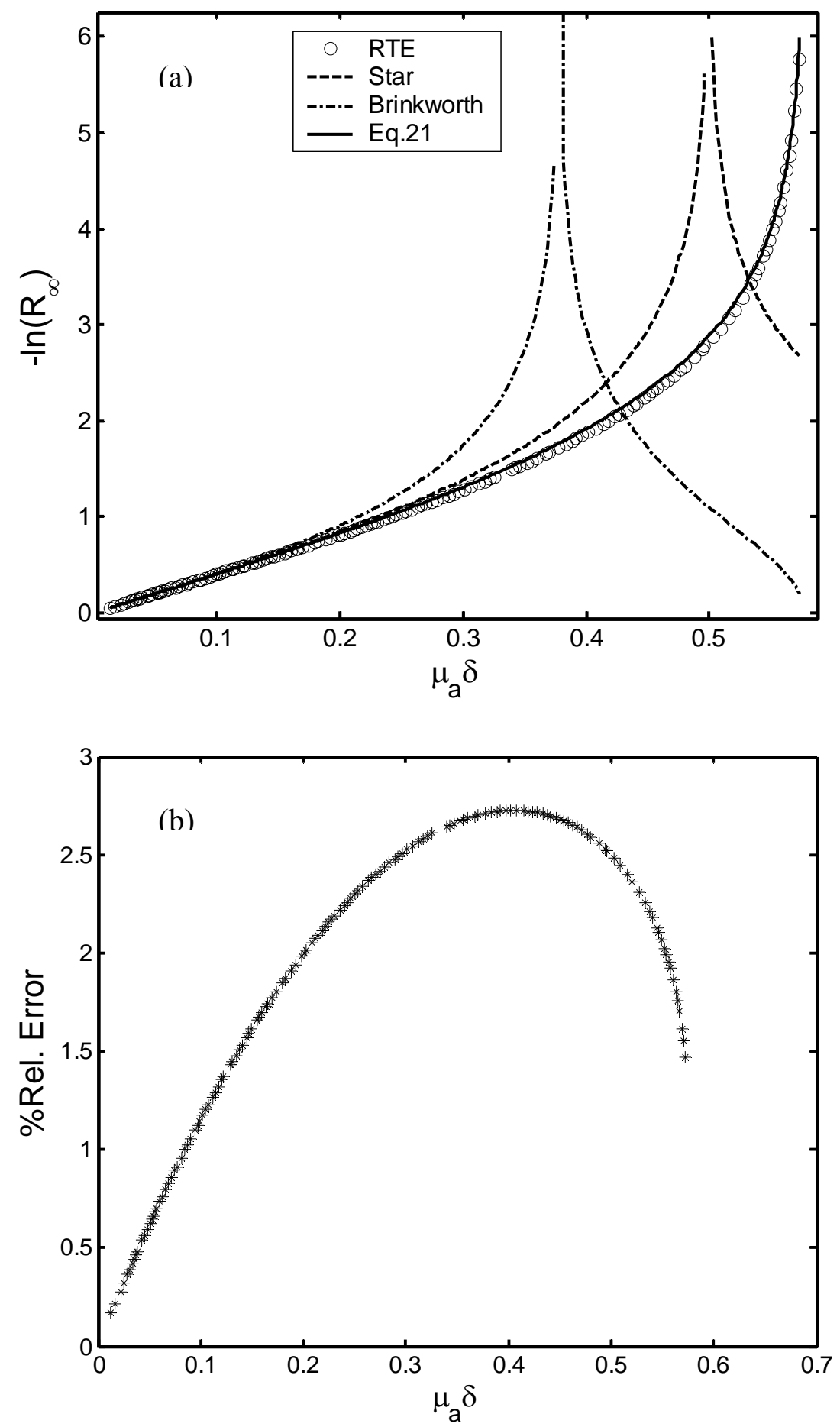

Figure3. Comparison of results for diffuse incident beam using K-M scattering coefficient $S$ computed with relationships given by Star et.al, Brinkworth and the present study. (a) $\mathrm{R}_{\infty}$ from the K-M equation using the various relationships is compared to the exact RTE. (b) Relative error of the K-M equation when (21) is used to related $\mathrm{S}$ with $\mu_{\mathrm{S}}^{\prime}$. 



Figure4. Comparison of results for collimated incident beam using K-M scattering coefficient $\mathrm{S}$ computed with (22) and (24). (a) $\mathrm{R}_{\infty}$ when $\mathrm{g}=0.1$; (b) $\mathrm{R}_{\infty}$ when $\mathrm{g}=0.9$; (c) Relative error when $\mathrm{g}=0.1$ and (d) Relative error when $\mathrm{g}=0.9$. 